\title{
Real Time Electrical Energy Monitoring and Cost Benefit Analysis using Smart Meter
}

\author{
Rajkumar Soni, Tushar Arora, Harshita Kumawat, Bhuveneshwari Dhamala, Himanshu Shekhar \\ Paliwal
}

\begin{abstract}
Energy is an essential component in supporting people's daily lives and is a significant economical element in development of the country. The eventual depletion of conventional energy resources and their harmful impacts on environment as well as the rising energy costs and the limitations of new energy resources and technologies have pushed efficient energy management to the top of the agenda. But how the energy utilization can be managed? A simple answer to this is viable and real time metering, which enables calculation of run time energy consumption and obtaining the real-time as well as cumulative cost. In this research an Innovative hardware and IoT based solution to this problem is availed that could provide live information related to consumption of electricity by various appliances. The methodology used in this research is mainly based on a hardware tool named Elite 440 which is a meter and provides the data about various electrical parameters. This data so obtained is made visible on the dashboard in a user friendly. The data so visible includes various parameters like voltage, current, power factor etc. Also the data so obtained on the dashboard gets updated in each five minutes and simultaneously the cost gets updated which makes it real time monitoring System.
\end{abstract}

Keywords: - MODBUS, Smart Metering, Cloud, Dashboard

\section{INTRODUCTION}

In today's era electrical energy is in the biggest demand and the research in this matter is at the peak. To remain competitive in today's market Industries consume electrical energy in bulk but face a very big problem of energy scarcity and hence escalated cost of the available energy. There are other alternate resources which produces electrical energy but they have their own limitation. So it has become a very big necessity for the consumers to reduce the losses in energy. But how it can be done?

The answer to this is real Time energy monitoring system that is the energy consumed must be metered first and the obtained data must be analyzed in order to find out where the energy losses are taking place and in what amount.

Revised Manuscript Received on March 5, 2020.

Rajkumar Soni*, Techno India NJR Institute of Technology Udaipur rajkumarsoni16@gmail.com

Tushar Arora, Techno India NJR Institute of Technology Udaipur Harshita Kumawat, Techno India NJR Institute of Technology Udaipur Bhuveneshwari Dhamala, Techno India NJR Institute of Technology Udaipur

Himanshu Shekhar Paliwal, Techno India NJR Institute of Technology Udaipur.
The conventional method to this is monthly or twice a month billing process which is very common and is a manual process but in this research an unconventional method of live data collection is presented in which one could see the data getting updated in each five minutes and the data so obtained is used to find the areas of energy losses and is also used for the future prediction of the energy consumption.

\section{ENERGY MANAGEMENT SYSTEM}

Energy is an essential component in supporting people's daily lives and is a significant economical element in development of the country. The eventual depletion of conventional energy resources and their harmful impacts on environment as well as the rising energy costs and the limitations of new energy resources and technologies have pushed efficient energy management to the top of the agenda. Energy management is the process of monitoring, controlling, and conserving energy in a building or organization. Also Energy management has become an important issue in recent times when many utilities around the world find it very difficult to meet energy demands which have led to load shedding and power quality problems.

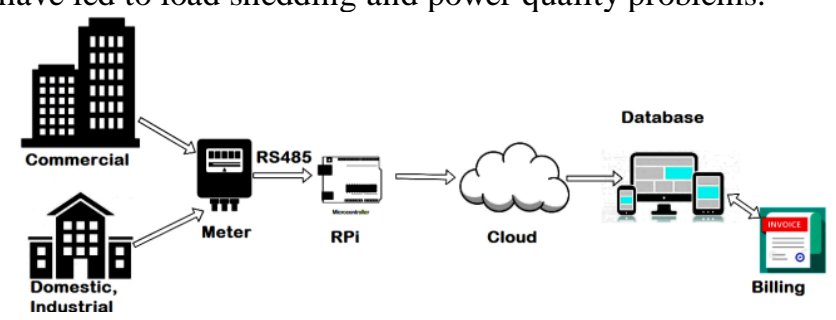

Fig. 1. Flow chart of smart metering. (figure caption)

The Energy- Saving Steps:

1. In order to save energy one need to meter the energy consumption and then collect the data in order to analyze it and to quantify the routine energy waste. One must also investigate the energy savings that could be made by replacing equipment.

2. Taking action to target the opportunities to save energy (i.e. tackling the routine waste and replacing or upgrading the inefficient equipment).

3. Tracking the progress by analyzing our meter data to see how well your energy-saving efforts have worked. 


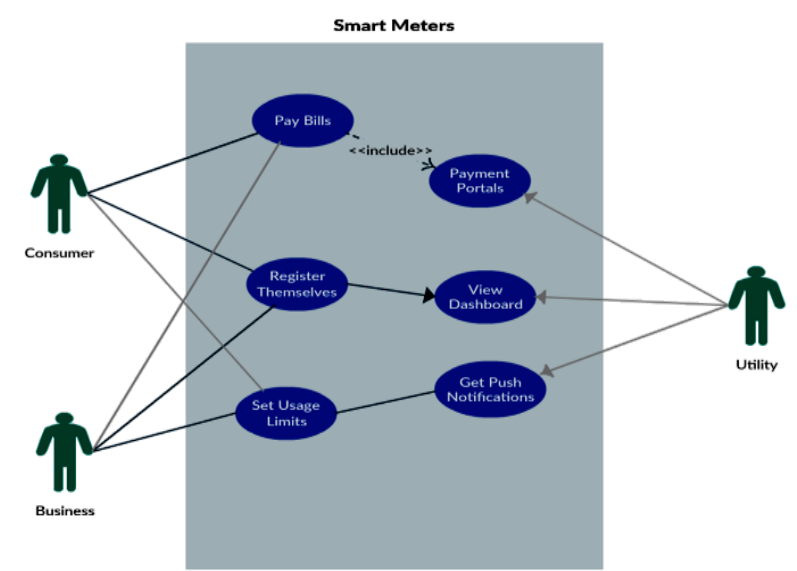

Fig. 2. Use Case for Consumers

Our research provides the facility for the consumer of live data collection which enables the user in analyzing the areas of energy losses and helps in managing the available energy.

\section{METHODOLOGY}

Our research mainly deals with finding out an innovative hardware and software based solution for Real Time Electrical Energy Monitoring system. To monitor the electrical energy here secure meters "Elite 440 " meter is used. Elite 440 meter is a multi-function panel meter with a multi-line digital display. This paper entails the solution of measuring and monitoring various electrical parameters. These meters are an ideal replacement for conventional energy meter, also they are substitute for energy meter provided by utility, in Industrial and Commercial connections. Elite 440 meters can work for High Voltage 3-phase 3-wire, High Voltage 3-phase 4-wire or Low Voltage 3 -phase 4 -wire and have accuracy of $1.0 \mathrm{~s}, 0.5 \mathrm{~s}$ and $0.2 \mathrm{~s}$.

The Elite 440 meter has the capability of Modbus communication so through Modbus communication protocol Raspberry pi 3 and the Meter is connected. The raspberry pi itself is an embedded computer, or a SBC (single board computer). This Raspberry Pi 3 is then connected with a network. This IoT device (Raspberry pi) fetches and reads the data from Elite 440 meter. This data includes present load state, voltage, current, power factor, frequency, time and various other electrical parameters which are pushed on the cloud server. This data gets updated in each five minutes since the load state is ON and the data so obtained is then stored on to the SQL database and is arranged into a systematic tabular form. This tabular format is then fetched through PHP, which is nothing but a general-purpose scripting language especially used in web development, and is displayed on the dashboard visible on the computer screen. This is how the real time energy is monitored and run time cost is calculated. Also through programming in PHP various Bar graphs are plotted which helps in predicting the future load.

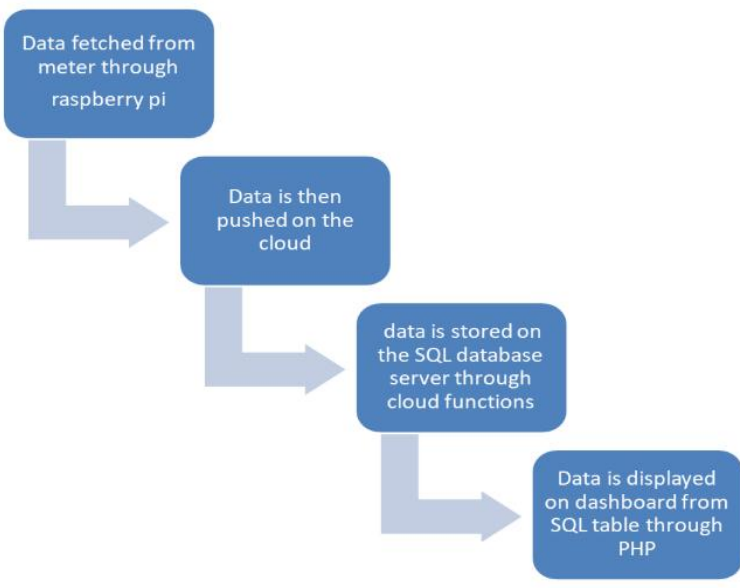

Fig. 3. Flow chart for Rpi and Smart meter

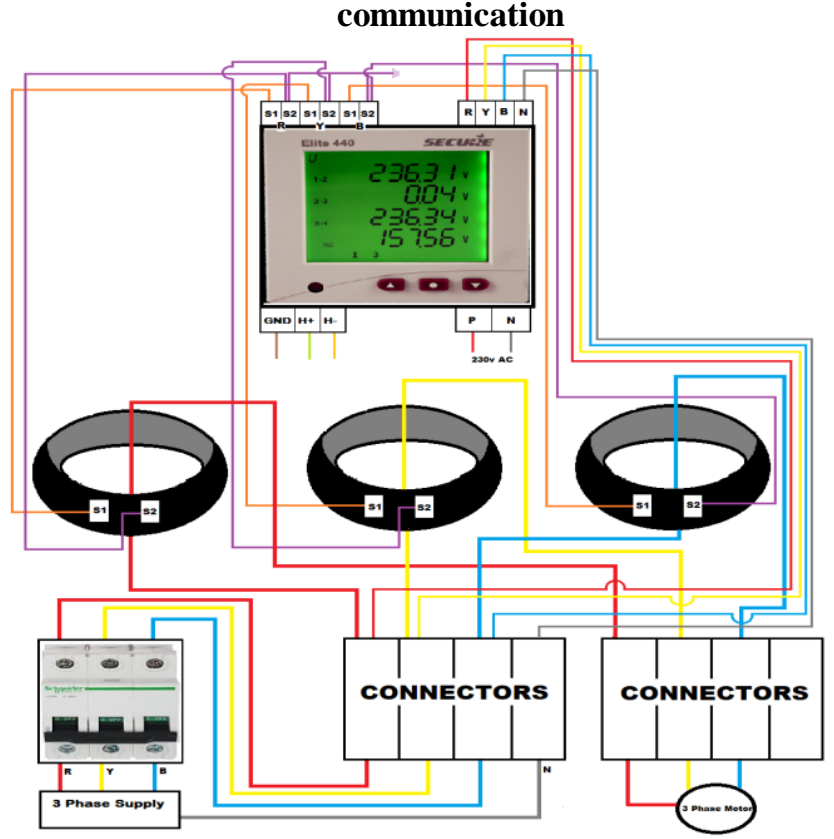

Fig. 4. Elite 440 connection diagram

Fig. 5. RS485 to USB converter for Rpi

Modbus communication protocol and Internet of Things:

Modbus communication between Smart meter and raspberry pi is used. The raspberry pi itself is an embedded computer, or also an SBC (single board computer). This smart meter consists of Raspberry Pi 3 connected with a network. This IoT device fetches and reads the data and pushes it on the cloud server. With the cloud function, the data is then stored on to the 
SQL database and arranged into a systematic tabular form which is then fetched through PHP and displayed on the dashboard.

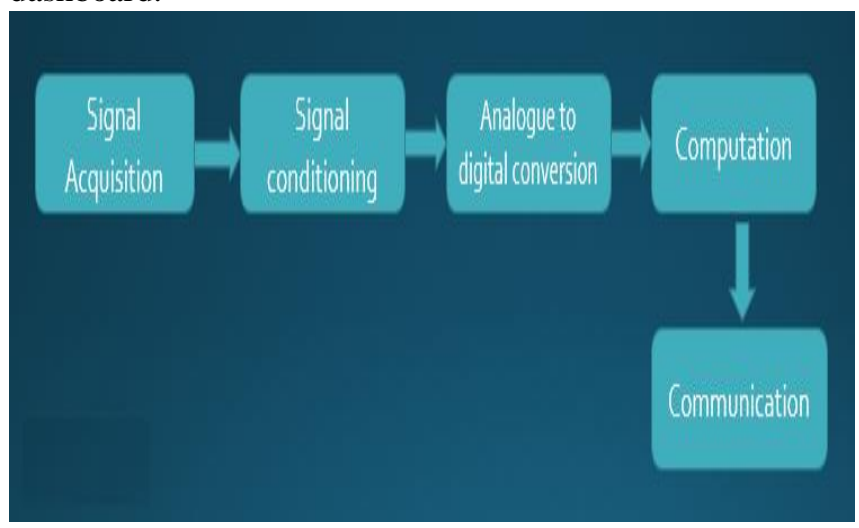

Fig. 6. Commnication Flow chart

Data Analytics:

Data analysis is a procedure of inspecting, rectifying, reforming the state and modeling data with the objective of identifying useful information, informing results, and supporting decision-making.

In few years when a sufficient amount of data is recorded model can be trained according to the readings so obtained so that prediction of the future readings can be done.

\section{PROBLEM STATEMENT}

This research is going to address the problem that Industries are facing and the problem is of energy scarcity which leads to increment in the cost of the same amount of energy being consumed. Due to the present competitive market energy consumption has become a very big challenge. It is very difficult for the industries to find out the areas of energy losses in the utility and hence decrease in the cost effectiveness of the whole system. The conventional method to this is monthly or twice a month billing process which does not even give the accurate data and one has to wait a lot to find out how much consumption machines have done. Also this is a manual process and chances of errors are more. Even using the conventional process it is not possible for the consumer to predict the future cost of the energy being consumed. Overall it can be mentioned that there is a need for a viable method for metering, calculating the energy consumption and obtaining the real-time as well as cumulative cost. Here in this research an Innovative hardware and IoT based solutions are presented that could provide live information related to consumption of electricity by various appliances.

There is a solution based on viable method for metering and calculating the energy consumption. Innovative software-based solutions that could provide live information related to consumption of electricity by various appliances. Methods for making it more cost-effective.

\section{RESULT AND DISCUSSION}

Fig. 1 shows the flow chart of smart metering, which simplifies the real time monitoring of electricity and unit billing. Fig. 2 shows that use case for consumers. Fig 3 shows the process of data accessing from Smart meter from Raspberry pi and sends it to cloud server. Fig. 4 shows the panel connection schematic diagram which consists of Secure make Elite 440 multi-function meter connected with C.T. (current transformers) of ratings 1000/20 C.T. ratio. And main MCB and this panel also shows the connection of load which is here three phase induction motor.

Fig. 5 shows the RS485 to USB converter which used here for communication of smart meter to Raspberry pi. Fig. 6 shows the signal acquisition to communication flow chart. Fig. 7 shows the hardware panel wiring of smart meter to Raspberry pi and load (three phase induction motor). Fig. 8 shows the smart meter Dashboard for real time monitoring of Parameters in which mainly shows the real time data based on load state ON or OFF, system Frequency, system Voltage, system Current, system Power Factor, Time and date. Fig. 9 shows the smart meter benefits for consumers like daily consumption, run time cost, power factor observation, prediction of future consumption and use limit of electricity in terms of units $\mathrm{kWh}$ can be set.

Fig. 10.shows the graph of electricity units $(\mathrm{kWh})$ versus time.

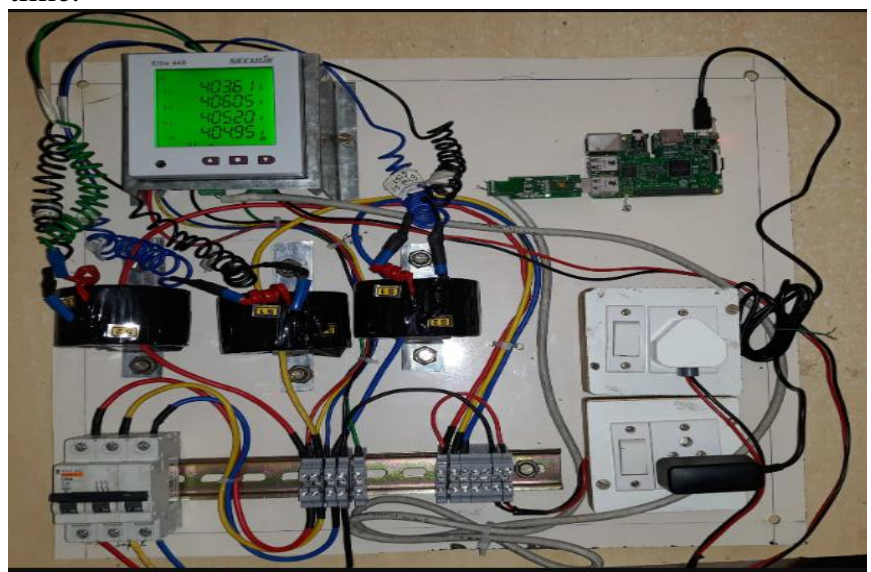

Fig. 7. Hardware smart meter panel

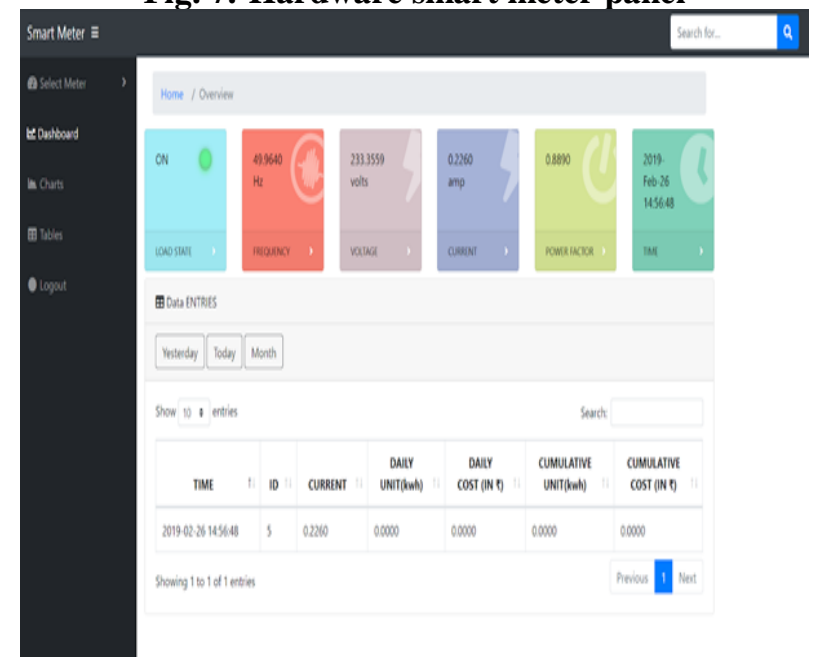

Fig. 8. Smart meter dashboard 
Real Time Electrical Energy Monitoring and Cost Benefit Analysis using Smart Meter

Fig. 9. Table I. Dashboard parameters for connected load

\begin{tabular}{|c|c|c|}
\hline Frequency & Unit (kWh) & Voltage \\
\hline 50.033001 & 0.31 & 236.554001 \\
\hline 49.995998 & 0.31 & 236.322845 \\
\hline 49.994999 & 0.31 & 235.369873 \\
\hline 49.988998 & 0.31 & 235.333766 \\
\hline 49.987 & 0.31 & 235.544617 \\
\hline 49.995998 & 0.31 & 236.376465 \\
\hline 50.014 & 0.31 & 235.204727 \\
\hline 50.047001 & 0.33 & 237.441803 \\
\hline 50.063999 & 0.33 & 237.453598 \\
\hline 50.056 & 0.33 & 237.374603 \\
\hline & \multicolumn{2}{|c|}{ Daily consumption } \\
\hline & & \\
\hline & \multicolumn{2}{|c|}{ Power factor observation } \\
\hline & \multicolumn{2}{|c|}{ Prediction of future consumption } \\
\hline iumer & \multicolumn{2}{|c|}{ Usage limit can be set } \\
\hline
\end{tabular}

Fig. 10. Smart meter advantages for consumers Authors should expect to be challenged by reviewers if the results are not supported by adequate data and critical details.

\section{CONCLUSION}

From the above obtained result it can be concluded that in order to save the energy and to find the areas of energy losses Energy monitoring is must and that can be done by the above mentioned methodology. Also cost benefit is a major advantage of energy management and hence when the real time data is available cost effectiveness increases since it becomes an easy a task to find out the loopholes in the present electrical system. One can get an idea about the future consumption by the available graphs on the dashboard. This paper entails the solution regarding:

- Eliminates manual meter readings by user

- Quick and accurate detailed readings

- Real time monitoring for consumers

\section{REFERENCES}

1. F. Abate et. al. "Smart Meter for the IoT", 2018 IEEE International Instrumentation and Measurement Technology Conference (I2MTC).

2. V. Preethi et. al. "DESIGN AND IMPLEMENTATION OF SMART ENERGY METER", 2016 International Conference on Inventive Computation Technologies (ICICT).

3. D.M. Lowe et. al. "Energy efficient induction motors performance characteristics and life cycle cost comparisons for centrifugal loads".

4. IEEE Trans. Industry Applications, vol. 33, no. 5, Sept./Oct. 1997, pp. $1312-1320$.

5. BSR Energy Management Handbook, April 2012.

6. Jixuan Zheng et. al. "Smart Meters in Smart Grid: An Overview", 2013 IEEE Green Technologies Conference (Genentech).

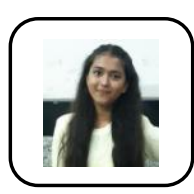

management", 2016 IEEE International Instrumentation and Measurement Technology Conference Proceedings.

8. A.Subba Rao et. al. "IOT Based Smart Energy Meter Billing Monitoring and Controlling the Loads", International Journal of Innovative Technology and Exploring Engineering (IJITEE) ISSN: 2278-3075, Volume-8 Issue-4S2 March, 2019.

9. The European Council for an Energy Efficient Economy (eceee) 2014.

10. P. O'Callaghan et. al. "Energy management: A comprehensive guide to reducing costs by efficient energy use", McGraw Hill, London, UK, 1992.

11. P. Kadurek et. al., "Theft Detection and Smart Metering Practices and Expectations in the Netherlands" Proc. IEEE PES Innovative Smart Grid Technologies Conf. Europe (ISGT Europe), pp. 1-6, 2010.

12. K. Hieta. V. Kao et. al. "Case Study of Smart Meter Deployment: Recommendations for Ensuring Ratepayer Benefits", March 2012.

\section{AUTHORS PROFILE}

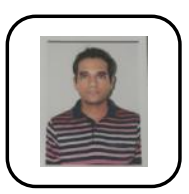

Rajkumar Soni received his B.Tech. (2012) in Electrical and Electronics Engineering from Rajasthan Technical Univ. and the M.Tech. (2019) in Energy and Power System from Sir Padampat Singhania Univ., Udaipur (Raj.), India $\mathrm{He}$ is currently working as an Assistant Professor in the Electrical Engineering Department at Techno India NJR Institute of Technology, College in Udaipur (Raj.)India. His research interests include Renewable Energy and Power System. He has also published research paper in EREECON 2014 on topic "Simulation of Vector Control of Induction Motor in MATLAB"

Email id: Rajkumarsoni16@gmail.com

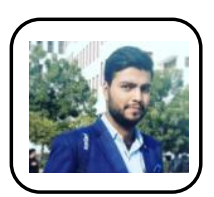

Tushar Arora is a student in the Electrical Engineering Department at Techno India NJR Institute of Technology, College in Udaipur (Raj.)India He will graduate with B.Tech. in the year 2021. He is the member of IEEE computer society and served ad volunteer and the host of the national workshop on IoT, IOTECH-2019 and he is an active member of Toastmasters international and is currently serving as president of Techno Ariston Toastmasters club. He is also awarded as a competent communicator and competent leader by Toastmasters international. His areas of interest includes Electrical Machines design and Electrical Power System. Other areas of interest includes public speaking and article writing. Email id: tushararoraudr@gmail.com

Harshita Kumawat is a student of Dept. of Electrical Engineering at Techno India N.J.R. Institute of Technology, Udaipur, and Rajasthan. She will graduate with B.Tech in the year 2021. She has served as volunteers in various technical and cultural events. Her Areas of Interests include Power Electronics and Energy Management System. Email id: harshitak546@gmail.com

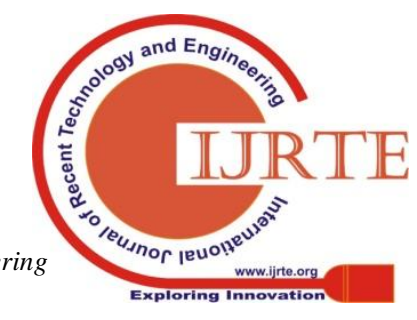


Bhuveneshwari Dhamala is a student of Dept. of Electrical Engineering at Techno India N.J.R. Institute of Technology, Udaipur, and Rajasthan. She will graduate with B.Tech in the year 2022. She has served as volunteers in various technical and cultural events. Her Areas of Interests include Electrical Machine and Electrical Circuit Analysis.Email id: bhuvidhamala@gmail.com

Himanshu Shekhar Paliwal is a student of Dept. of Electrical Engineering at Techno India N.J.R. Institute of Technology, Udaipur, and Rajasthan. He will graduate with B.Tech in the year 2022. He has served as volunteers in various technical and cultural events. His areas of Interests include Electrical Machine and Electrical Circuit Analysis. Email id: himanshuvishnu12345@gmail.com 